\title{
Transceiver error reduction by design prototype system based on neural network analysis method
}

\author{
Muthna Jasim Fadhil, Maitham Ali Naji, Ghalib Ahmed Salman \\ Departement of Electrical Power Technical Engineering, Electrical Engineering Technical College, \\ Middle Technical University (MTU), Iraq
}

\begin{tabular}{l} 
Article Info \\
\hline Article history: \\
Received May 2, 2019 \\
Revised Jul 7, 2019 \\
Accepted Sep 26, 2019 \\
\hline Keywords: \\
Encoder and decoder feature \\
Forward neural network \\
MATLAB \\
Self organizing feature map \\
Spiking neural network
\end{tabular}

Spiking neural network

\begin{abstract}
Code words traditional can be decoding when applied in artificial neural network. Nevertheless, explored rarely for encoding of artificial neural network so that it proposed encoder for artificial neural network forward with major structure built by Self Organizing Feature Map (SOFM). According to number of bits codeword and bits source mentioned the dimension of forward neural network at first then sets weight of distribution proposal choosing after that algorithm appropriate using for sets weight initializing and finally sets code word uniqueness check so that matching with existing. The spiking neural network (SNN) using as decoder of neural network for processing of decoding where depending on numbers of bits codeword and bits source dimension the spiking neural network structure built at first then generated sets codeword by network neural forward using for train spiking neural network after that when whole error reached minimum the process training stop and at last sets code word decode accepted. In tests simulation appear that feasible decoding and encoding neural network while performance better for structure network neural forward a proper condition is achieved with $\gamma$ node output degree. The methods of mathematical traditional can not using for decoding generated Sets codeword by encoder network of neural so it is prospect good for communication security.
\end{abstract}

Copyright (ङ 2020 Institute of Advanced Engineering and Science. All rights reserved.

\section{Corresponding Author:}

MuthnaJasimFadhil,

Departement of Electrical Power Technical Engineering,

Electrical Engineering Technical College,

Middle Technical University (MTU), Baghdad, Al-Doora, Iraq.

Email: muthnafadhil@gmail.com

\section{INTRODUCTION}

The process of communication reliability improve using encoding and decoding in general, bits check to bits source relation adopted in mentioned algorithm. For example code block linear in typical bits codeword and bits source relationship is complete for matrix generating or matrix check, in other words depending on matrix check (expression check) and bits source produced bits check [1]. The correction of error applied when expression check mismatch with bits codeword received in processing decoding where codes as LDPC, Codes product turbo and codes convolution is codes traditional decoded in method novelty adopted in general neural network. Decoder neural network two kinds, firstly decoder major architecture adopted in neural network and secondly decoder in role supportive adopted in neural network. spiking neural network (SNN) is typical decoder neural network solution adopted generally so that advance sets codeword using for adequately trained decoder. Channel multipath fading Rayleigh dispersive highly over transmitted information decode and equalize jointly used neural network of Hopfeild [2]. Investigated method of decoding exist optimizations depending on recurrent neural network(RNN)unsupervised. Proposed algorithm decoding corresponding and scheme extraction information extrinsic depending on neural network function bases radial. Implementation of high speed computation in parallel and real time implement for neural 
network proposed by decoder code block linear [3]. Neural network functions activations and weights should be changed when transaction with Field Galois non-binary so that several bits error detection and correction. Approach quantization vector robust based SOM decoding soft which has suitable communications Wireless Proposed neural network based on decoder convolution novelty where technique decoding turbo traditional less performance than it. SSOM form presented decoder neural network general novelty using for rules encoding regardless time for decoding and learning achieved [4]. Code traditional decoding is mainly decoding neural network study, encoding of neural network is researchers concerned rarely beside bits source original encode by adopted neural network, the bits codeword and bits source relationship express in method novelty [5]. Encoding neural network study is the first stage, this paper achieved various contributions, such as firstly encoding and decoding neural network realize which proposed is solution workable, secondly method mathematic traditional cannot using for describe encoding neural network rule because contain feature confidential certain and finally method of mathematic traditional cannot using to decoded sets of codeword that produced by decoder neural network beside suitable system for communication security [6].

\section{ENCODER NEURAL NETWORK A CHIEF IDEA}

\subsection{Encoder Traditional Mechanism}

The majority difference of encoder traditional from encoder of neural network is sets codeword mechanism generation. Encoder and decoder traditional archticture shows in Figure 1 [7-9]. For encoder traditional bits codeword $\mathrm{M}+\mathrm{F}$ generated by using bits source original $\mathrm{M}$ via matrix generation where bits check redundancy bits $\mathrm{F}$ included so that process of decoding matrix check using for check bits codeword $\mathrm{M}+\mathrm{F}$. Actually, encoder traditional core is matrix generating where speaking generally beside encoder traditional select various bits source to determine desired bit check depending on matrix generating or matrix check [10, 11]. For example graph bipartite sparse using for build up codes LDPC as shown in Figure 2 which represent different form of matrix check by means any bit check is determined by bit specific. Figure 2 shows M3 bit check is determined by the following expression:
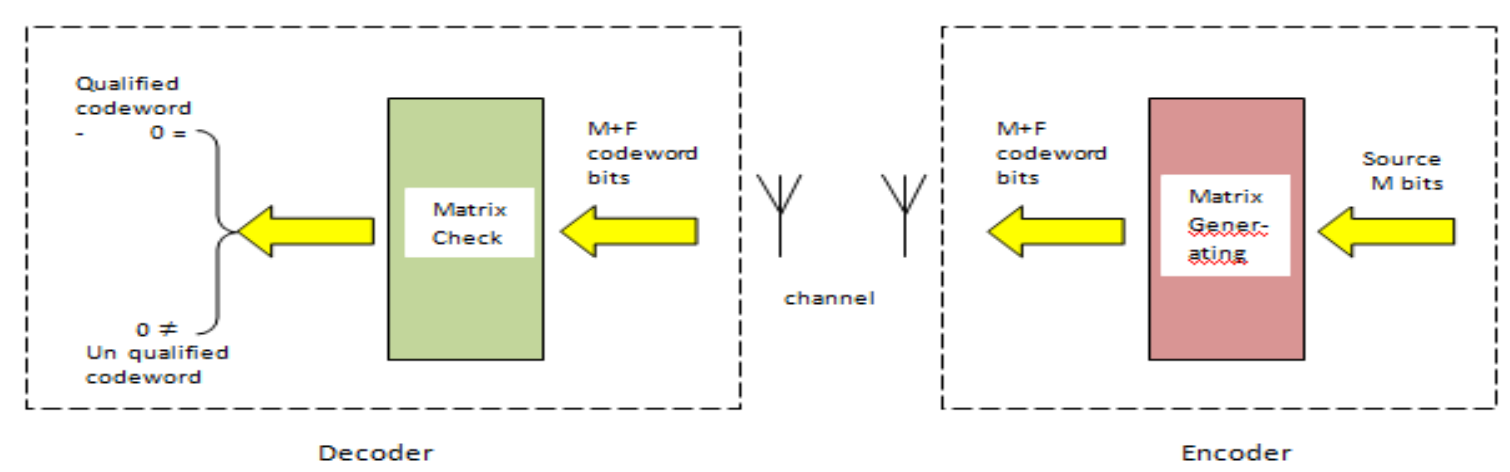

Figure 1. Encoder and decoder traditional architecture

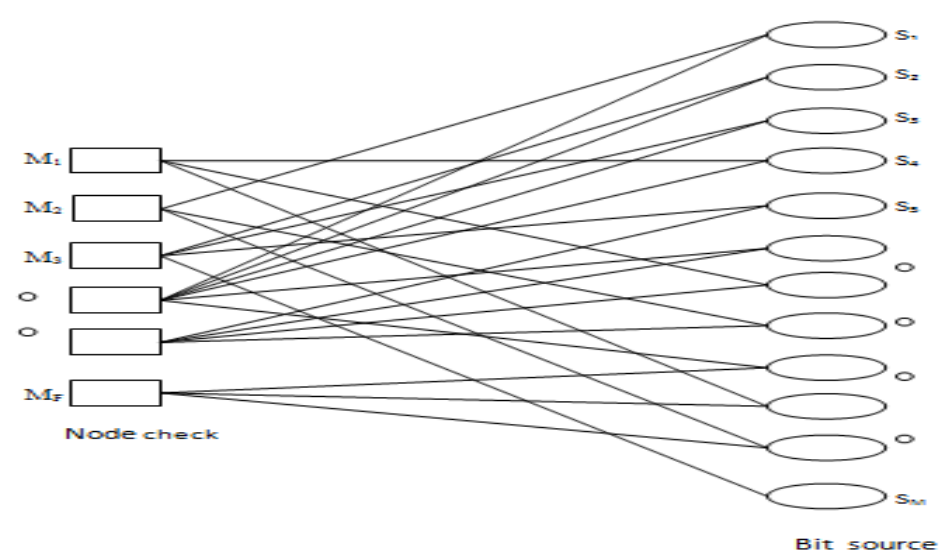

Figure 2. Codes LDPC graph bipartite sparse 


$$
\mathrm{M}_{3}=\mathrm{S}_{2} \oplus \mathrm{S}_{3} \oplus \mathrm{S}_{5} \oplus \mathrm{S}_{\mathrm{M}}
$$

Encoders traditional has various characteristics, in sum:

a) Bits codeword are produced by rules or formulas specific.

b) Bits source original have relationship with bits codeword.

c) Various rules of producing bits codeword mean various type code [12-14].

\subsection{Encoder Neural Network Mechanism}

Neural network generated results producing sets codeword as encoder neural network, encoder and decoder neural network architecture shows in Figure 3.

a) This encoder comparing with encoder traditional has two majority aspects different:

b) Bits codeword produced by neural network forward beside formulas specific or matrix generating.

c) Type code calculated by sets weight own and its neural network generating.

Neural network forward is selected to generate bits codeword M+F from bits source $\mathrm{M}$ because it has same function of matrix generating in encoder traditional [15-17]. Neural network forward also known as neural network generating difference from neural network normal by some specific such as SNN,SOFM,etc. Sets codeword way generated by neural network forward as shown in Figure 4 [18-20].

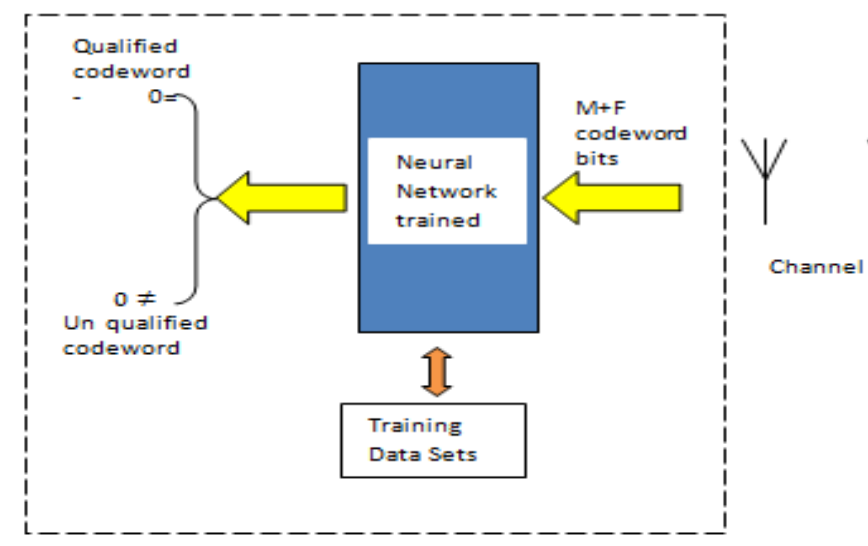

Decoder

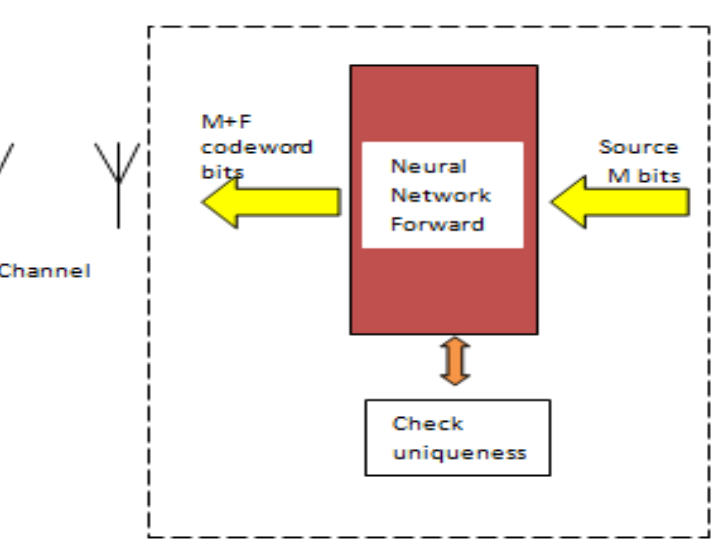

Encoder

Figure 3. Encoder and decoder neural network architecture

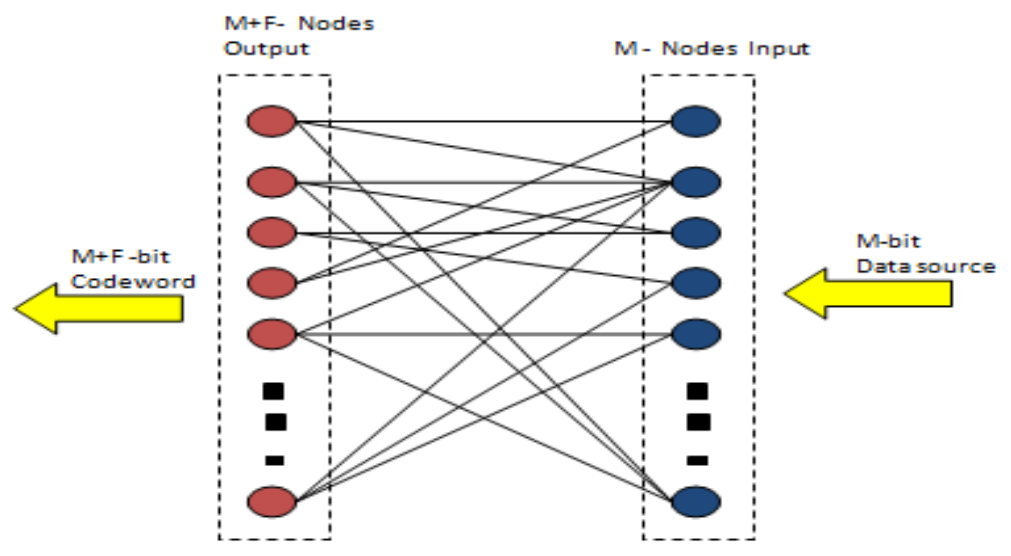

Figure 4. Encoder neural network forward architecture

Encoder architecture is same of neural network SOFM and the difference compare with neural network SOM traditional is: 
a) Only one way process forward for neural network generating by means that has not process learning and competition.

b) Neural network forward doesn't connected fully, it means that nodes input partly connected to each node output through weight beside whole model connection.

c) Neural network forward range certain should be include range sets weight value so that goals to result guarantee node output with permitted scope.

d) Neural network SOFM traditional difference from node output structure as shown in Figure 5 [21, 22].

Figure 5 shows that Qi calculated by $\mathrm{Nj}$ corresponding and Hij weight connected and $\mathrm{f}()$ is a function decision which calculated the result. As shown in (3) using to determine $\mathrm{Z}_{\epsilon}$ which represent function decision of sets weight threshold.

$$
\begin{aligned}
& Q_{i}=f\left(\sum_{j=1}^{M} N_{i} * H_{i j}\right)=\left\{\begin{array}{ll}
1 & Q_{i}>Z_{\epsilon} \\
0 & Q_{i}<Z_{\epsilon}
\end{array} i=1,2, \ldots . M+F\right. \\
& \mathrm{H}_{\mathrm{ij}}=\left\{\begin{array}{c}
\mathrm{H}_{\mathrm{ij}} \text { connected node input } \mathrm{j} \text { and node output } \mathrm{i} \\
0 \text { not connected node input } \mathrm{j} \text { and node output } \mathrm{i}
\end{array}\right. \\
& \mathrm{Z}_{\epsilon}=\overline{\sum_{\mathrm{j}=1}^{\mathrm{M}} \sum_{\mathrm{i}=1}^{\mathrm{M}+\mathrm{F}} \mathrm{N}_{\mathrm{j}}} * \mathrm{H}_{\mathrm{ij}}
\end{aligned}
$$

Weight range must be in range mentioned as (4).

$$
\begin{aligned}
& \mathrm{H}_{\mathrm{ij}}<\mathrm{H}_{\epsilon} \mathrm{H}_{\epsilon}=\frac{1}{\gamma(\mathrm{M}+\mathrm{F})} \sum_{\mathrm{j}=1}^{\mathrm{M}} \sum_{\mathrm{i}=1}^{\mathrm{M}+\mathrm{F}} \mathrm{S}_{\mathrm{ij}} \\
& \mathrm{S}_{\mathrm{ij}}=\left\{\begin{array}{l}
1 \mathrm{H}_{\mathrm{ij}} \neq 0 \\
0 \mathrm{H}_{\mathrm{ij}}=0
\end{array}\right.
\end{aligned}
$$

$\gamma$ is degree average of node output in neural network forward which is nodes output connected to sets weight average number. Speaking generally, $\gamma$ is calculated by (6) when $\gamma$ equal M+F, sets weight connected fully to forward neural network, that is means all nodes input have relationship with each node output.

$$
\gamma=\frac{\delta \mathrm{M}}{(\mathrm{M}+\mathrm{X})}
$$

$\delta$ is the node input degree represent nodes output related number, high $\delta$ means high responsibility to node input individual so that process encoding computing more complexity results [23-25].

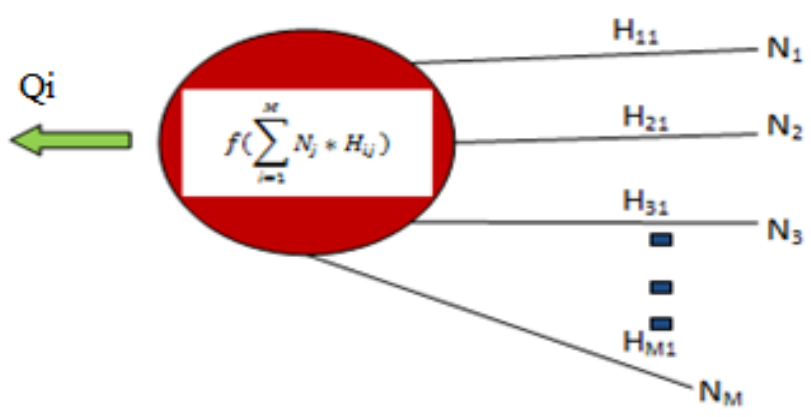

Figure 5. Node output structure

\subsection{Encoder Neural Network Steps Specification}

Encoder neural network can be achieved as following:

a) Depending on $\gamma$ neural network constructed mentioned bits codeword and bits source dimention.

b) Number random in group using for sets weight initializing.

c) Bits source accept and bits codeword calculate depending on (1)-(6).

d) Jump instruction using for bits source next accept to step (3).

e) Sets codeword uniqueness check. 
Figure 6 following describe neural network forward constructing algorithm where $\mathrm{i}$ node input degree is $\delta_{\mathrm{i}}$ and $\mathrm{j}$ node output degree is $\gamma_{\mathrm{j}}, \mathrm{H}_{\mathrm{ij}}$ is tag weights where stage initializing $\mathrm{H}_{\mathrm{ij}}$ corresponding for multiply used and zero is $\mathrm{H}_{\mathrm{ij}}$ value default. Uniqueness checking algorithm shows in Figure 7 . Where $\sigma$ is rate learning, bit input specific impact strengthen by sets weight related increasing and $\partial$ (rate suppressing)using for bit output related impact reduce by sets weight related decreasing, in general $\partial$ rate suppressing more smaller than $\sigma$ rate learning.

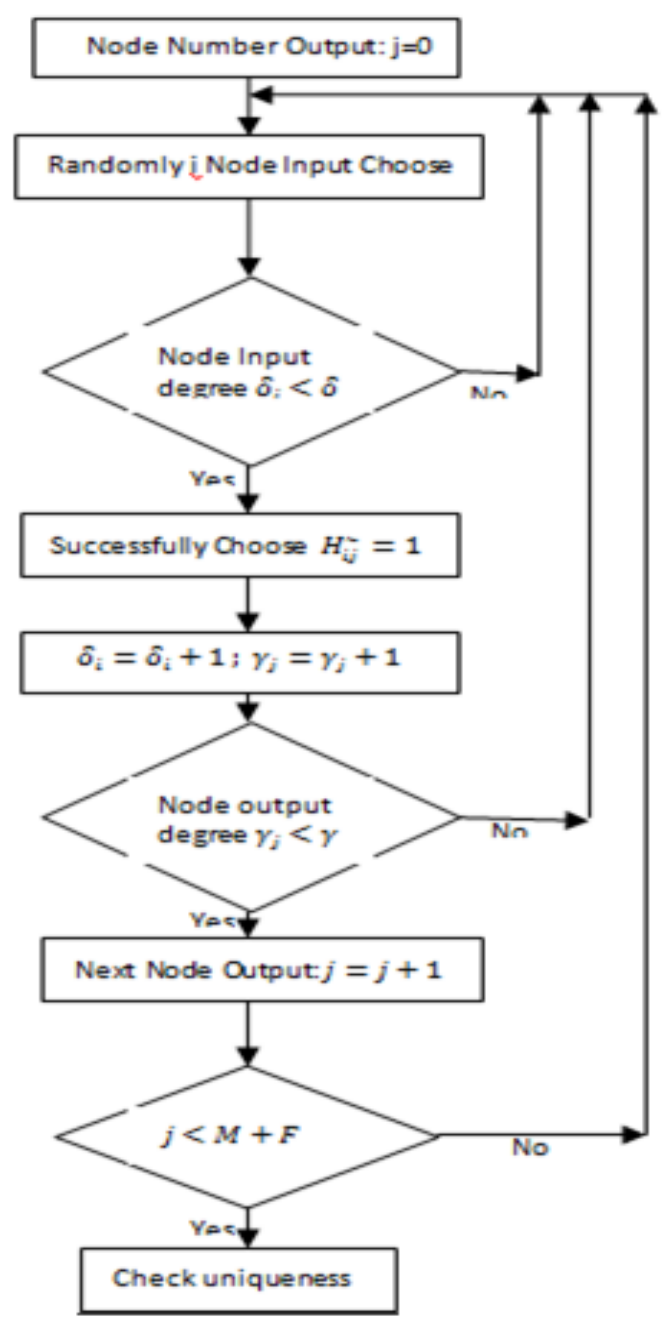

Figure 6. Neural network forward constructing algorithm

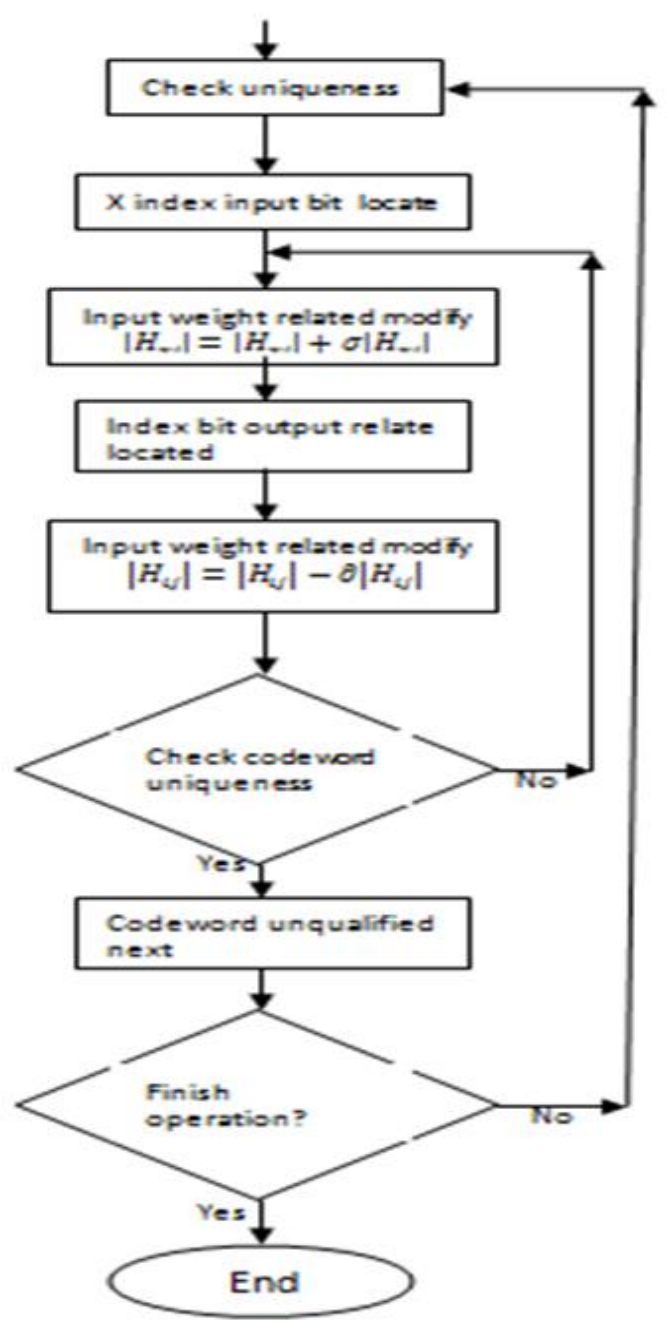

Figure 7. Uniqueness checking algorithm

\section{DECODER NEURAL NETWORK DESIGN}

\subsection{Decoder Neural Network Solution}

Traditional way for decoding can not apply in generated codeword by encoder Neural Network forward because cannot using expression or matrix generating to describe bits source relationship with bits codeword then mathematical traditional expression can not using for encoder neural network forward codeword generated rule. At last rules encoding recorded in box block encoder of neural network forward where the box block neural network forward algorithm and structure is sets weight. so that encoder neural network that generated sets codeword decoded by decoder built in neural network. Find symmetric method for function encoding achieve SNN, SNN decoder architecture as shown in Figure 8, therefore find two stages for decoder :stages decoding and training. For stage training, sets sample codeword group using for trained SNN where neural network forward generated bits codeword and bits source using for constructed the above group while for stage decoding the results output ultimate calculations and bits codeword accepts by SNN trained. 


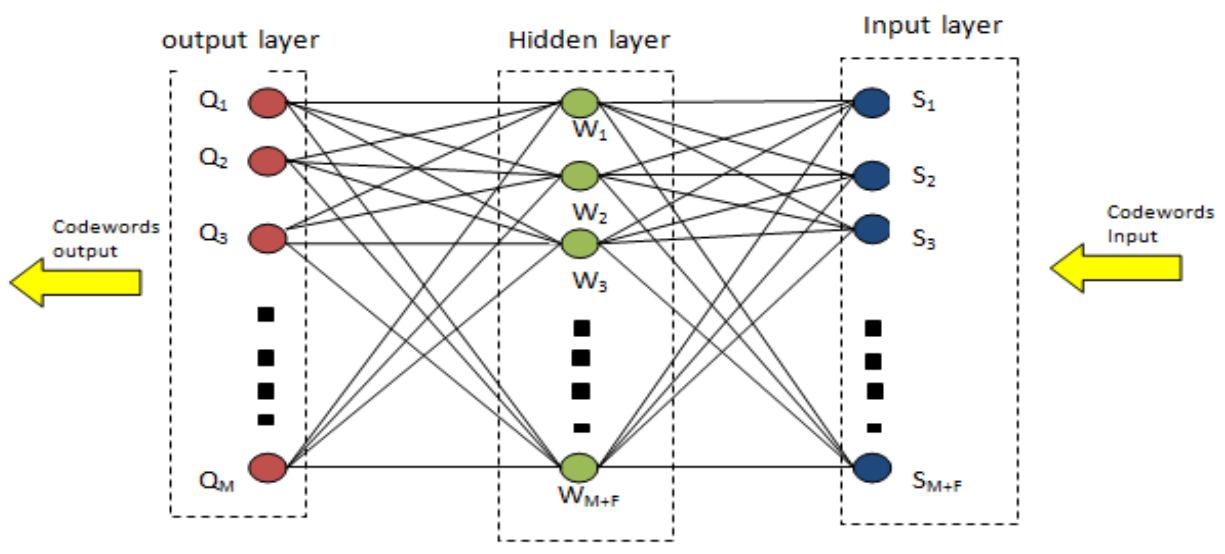

Figure 8. Decoder SNN architecture

\subsection{Decoder Neural Network Steps Specification}

Decoder neural network stages are stages decoding and training, bits source and bits codeword composed in stage of training where sets sample training using for process of learning, the following describe these steps specific.

a) Neural network forward (encoder) using to produce sets data codeword training.

b) Depending on sets sample training size SNN constructed.

c) Algorithm specific using for SNN training so that convergence reach. Beyond stage training, bits codeword decoding by decoder adopts by SNN which is considered decoder traditional in stage of decoding.

d) One by one layer result calculation and bits codeword accepted.

e) SNN layer output (decoder result) export.

f) Step 1 jump and bits codeword next accepted.

\section{RESULTS AND ANALYSIS}

The results of this work explored in this section, various $\gamma$ (Cama) comparison BER: in Figure 9 reached conclusions that increases SNR when decreases BER and change $\gamma$ when fluctuates BER. SNR same of $\gamma$ proper choosing using to obtained BER good beside computation complex more means $\gamma$ is high so system needed a lot of resource in this condition and (6) using for generated $\gamma$ proper.

Failure check uniqueness possibility available by means bits codeword $\mathrm{M}+\mathrm{F}$ not only depending on bits source M so that failure has two factors: i. Sets codeword current not suitable neural network structure. ii. Must be modified sets weight so that matching sets codeword. The problem solve check uniqueness algorithm depending on (2) where sets weight modification by guaranteed uniqueness, encoder uniqueness guaranteed structure neural network modification construct in future study.

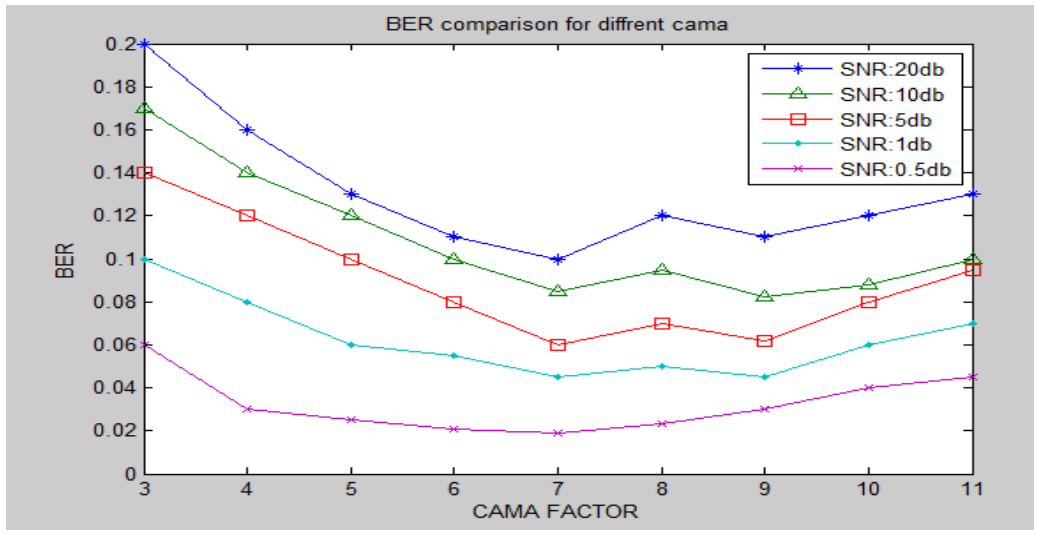

Figure 9. Various $\gamma$ comparison with BER

Transceiver error reduction by design prototype system based on neural network... (Muthna Jasim Fadhil) 
Table 1 shown data test model 6 layers matrix confusion where data test coherence temporal considering with accuracies classification. Matlab simulation using to represent this situation as shown in Figure 10 the best results got in layer6 where it reach the accuracy to $1(100 \%)$ at 34.38 ms. Figure 11 represent the comparison this work with other related works, the results obtained clearly explain that when applied algorithm SOFM-SNN for encoding and decoding process got better results.

Table 1. Dataset Testing best Model(model 6 layers) Matrix Confusion

\begin{tabular}{ccccccc}
\hline Time & \multicolumn{6}{c}{ Accuracies on different labels } \\
$(\mathrm{ms})$ & Layer 1 & Layer 2 & Layer 3 & Layer 4 & Layer 5 & Layer 6 \\
\hline 7.92 & $87 \%$ & $88 \%$ & $90 \%$ & $93 \%$ & $94 \%$ & $97 \%$ \\
16.52 & $90 \%$ & $91 \%$ & $92 \%$ & $93 \%$ & $94 \%$ & $98 \%$ \\
24.7 & $91 \%$ & $93 \%$ & $94 \%$ & $94 \%$ & $96 \%$ & $99 \%$ \\
34.38 & $91.5 \%$ & $94 \%$ & $96 \%$ & $95 \%$ & $96 \%$ & $100 \%$ \\
43.65 & $92 \%$ & $95 \%$ & $97 \%$ & $97 \%$ & $98 \%$ & $100 \%$ \\
51.97 & $93 \%$ & $96 \%$ & $97 \%$ & $97 \%$ & $98 \%$ & $100 \%$ \\
59.22 & $94 \%$ & $97 \%$ & $98 \%$ & $98 \%$ & $98 \%$ & $100 \%$ \\
\hline
\end{tabular}

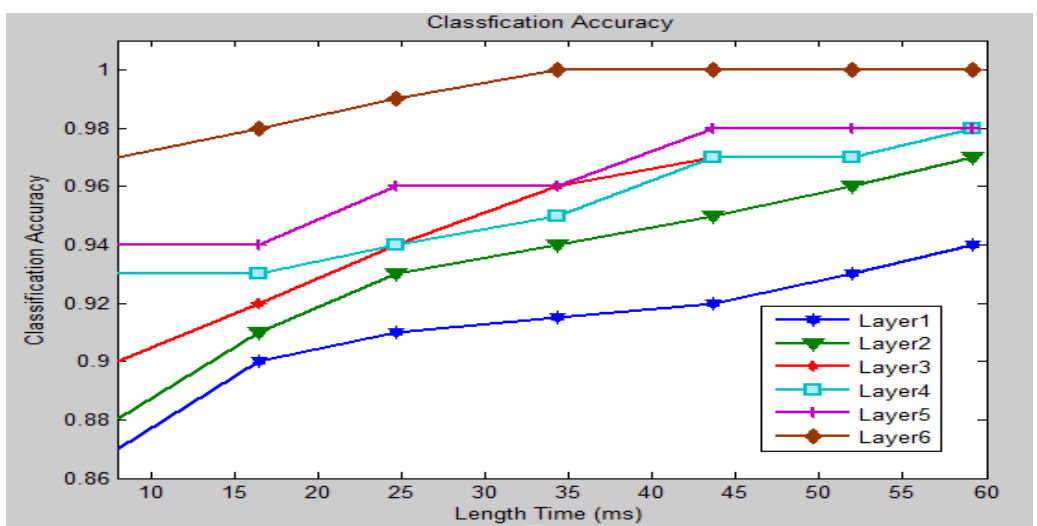

Figure 10. Length time different dataset accuracies classification

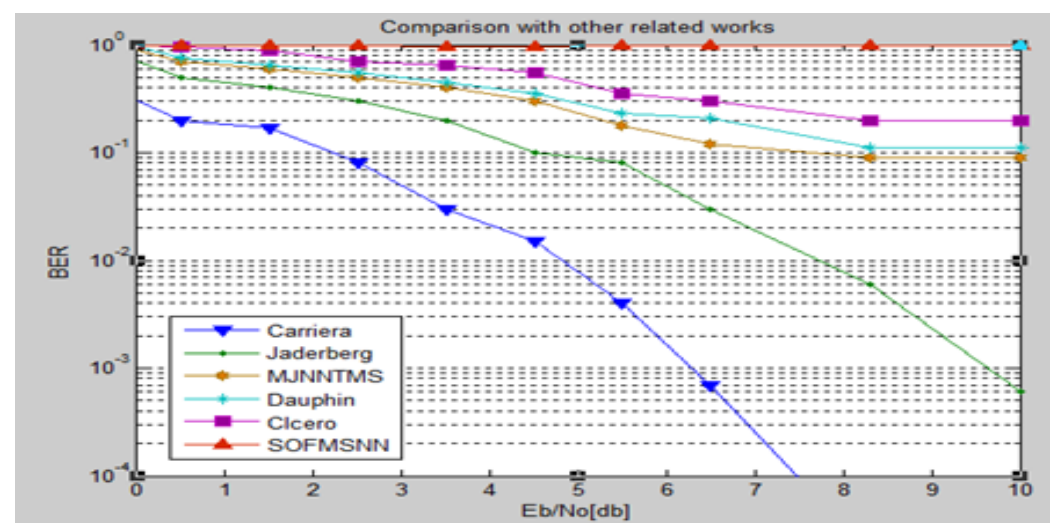

Figure 11. Comparison this work with other related works

\section{CONCLUSION}

Future security of Coding channel is encoding neural network, sets weight and itself neural network encoding rule whole so that method mathematic traditional described disable where a block box such algorithm encoding. Neural network adopted by encoding and decoding together. Better results got when applied this algorithm in this clustering of SOFM-SNN encoding and decoding and that is clear in Table1 when compared this work with other related works. Way of distributed using in neural network to stored encoding and decoding rule specific additional that neural network mode parallel cause greatly speed and reliability increasing where application future prospected desire. 


\section{REFERENCES}

[1] S.M. Berber and V. Kecman, "Convolutional decoders based on artificial neural networks", IEEE International Joint Conference on Neural Networks-Proceedings, vol. 2, pp:1551-1556, 2004.

[2] S.M. Berber, "Soft output decision convolutional (SONNA) decoders based on the application of neural networks," Engineering Applications of Artificial Intelligence, vol. 21, no. 1,pp. 1-13, 2008.

[3] J. Carreira et al., "Action Recognition?A New Model and the Kinetics Dataset". In IEEE Conf. on Computer Vision and Pattern Recognition, 2017.

[4] M. Jaderberg, et al.., "Spatial transformernetworks", In Advances of Neural Information Processing Systems, pp. 2017-2025, 2015.

[5] E. Coersmeier et al.,"Combining Cognitive Radio andSoftware Radio Approach for Low Complexity Receiver Architecture", Proc. of the Int. Sym. on Advanced Radio Technologies (ISART), 2007.

[6] T. N. Sainath, et al.,"Deep convolutional neural networks for LVCSR," In Proc. IEEE Int. Conf. Acoust., Speech, Signal Process. (ICASSP), pp.8614-8618, May 2013.

[7] C.Santos and M. Gatti. "Deep Convolutional Neural Networks For Sentiment Analysis Of Short Texts". In Proceedings of the 25thInternational Conference on Computational Linguistics (COLING), Dublin, Ireland, 2014.

[8] Y.Dauphin, et al.,"Language modeling with gated convolutional networks", arXiv preprint arXiv:1612.08083, 2016.

[9] Q Wu, et al., "Multi-objective optimization of a distributed energy network integrated with heating interchange", Energy, vol.109, pp.353-364,2016.

[10] Upendar, et al.," PSO and ANN-based fault classification for protective relaying". Iet Generation Transmission \& Distribution, vol.4, pp.1197-1212, 2010.

[11] C.Mariela, et al., "Multi-stage feature selection by using genetic algorithms for fault diagnosis in gearboxes based on vibration signal". Sensors, vol.15,pp. 23903-23926, 2015.

[12] A. zadeha, et al.,"A flexible algorithm for fault diagnosis in a centrifugal pump with corrupted data and noise based on ANN and support vector machine with hyper-parameters optimization”, Applied Soft Computing, vol.13, pp. 1478-1485,2013.

[13] J. Barton, et al.," A Miniaturized Modular Platform for Wireless SensorNetworks", Proc., European Conf. on Circuit Theory and Design,pp. 35-38, 2005.

[14] M.Fadhil and M. Farhan," Design a prototype FPGA model for target detection by radars passive based on synthetic aperture radar algorithm", Journal of Engineering Science and Technology, vol. 14, no. 3,pp. 1542-1557, 2019.

[15] A.Sarkar, et al.,"Multilayer neural network synchronized secured session key based encryption in wireless communication", Indonesian Journal of Electrical Engineering and Computer Science (IJEECS), Vol. 14, No. 1, pp. 169-177, 2019.

[16] F. Pardede, et al., "Convolutional Neural Network and Feature Transformation forDistant Speech Recognition", International Journal of Electrical and Computer Engineering (IJECE), Vol.8, No.6, pp. 5381-5388,2018.

[17] H.Rahimi and M.Zolfaghari,"New Model Reference Adaptive System Speed Observer for Field-Oriented Control Induction Motor Drives Using Neural Networks", Bulletin of Electrical Engineering and Informatics (BEEI), Vol. 5, No. 1, pp. 25-36, March 2016.

[18] T. Dewi, et al., "Neural Network Controller Design for a Mobile Robot Navigation; a Case Study", Proc. EECSI 2017, Yogyakarta, Indonesia, pp.19-21 September 2017.

[19] L.Eng, et al., "Distance weighted K-Means algorithm for center selection in training radial basis function networks", IAES International Journal of Artificial Intelligence, Vol. 8, No. 1, pp. 54-62, 2019.

[20] T.Gibet, et al., "Comparative Study of Neural Networks Algorithms for Cloud Computing CPU Scheduling", International Journal of Electrical and Computer Engineering (IJECE), Vol.7, No.6, pp. 3570-3577, December 2017.

[21] H.Nugroho, et al., "Deep Learning for Tuning Optical Beamforming Networks", TELKOMNIKA Indonesian Journal of Electrical Engineering and Computer Science, Vol.16, No.4, pp. 1607-1615, 2018.

[22] C.Hong, et al., "Progress in neural network based techniques for signal integrity analysis-a survey", Bulletin of Electrical Engineering and Informatics, Vol.8, No.1, pp. 276-282, 2019.

[23] N.Thuy, et al., " New Version of Adaptive Speed Observer based on Neural Network for SPIM", International Journal of Power Electronics and Drive System (IJPEDS), Vol.9, No.4, pp.1486-1502, 2018.

[24] M.Fadhil, et al., "Design and implementation a prototype system for fusion image by using SWT-PCA algorithm with FPGA technique", International Journal of Electrical and Computer Engineering (IJECE), Vol.10, No.1, pp. 757-766, 2020.

[25] A.Azamimi, et al., "Cervical cancer detection method using an improved cellular neural network (CNN) algorithm", Indonesian Journal of Electrical Engineering and Computer Science, Vol.14, No.1, pp. 210-218, 2019. 